\title{
Quantitative Assessment of Probe and Spectrometer Performance for a Multi-Channel CCD-based Fiber Optic Dissolution Testing System
}

Guy W. Inman

Principal Consultant

Automation Resources

5216 Hough Rd., Hillsborough, NC 27278 email : inmangw@bellsouth.net
Introduction

A mong the important factors that must be addressed for successful application of in situ UV fiber optic dissolution testing are spectrometer and probe performance. The technological challenge to system developers has been to replace automated or manual sample removal, filtration, and off-line analysis with effective on-line, in-vessel analysis while meeting regulatory requirements. Critical differences between conventional and in situ technologies are related to multiple sample spectral acquisition, multiple sampling time constraints, a significant reduction in the amount of available light signal due to fiber optics, lack of filtration, and replacement of the cuvette with a fiber optic probe. The purpose of this paper is to assess critical fundamental system spectrometer and probe related performance parameters for a multi-channel spectrometer system (OPT-DISSTM,LEAPTechnologies, Inc., Carrboro, NC) consisting of a CCD array detector and low-displaced-volume $\mathrm{ARCH}^{\mathrm{TM}}$ probes.

\section{Methods}

\section{Materials}

USP grade distilled and de-ionized water was used in all test solutions. Linearity test solutions were prepared from NIST 935a potassium dichromate standard reference material dissolved in $0.01 \mathrm{~N}$ sulfuric acid. Stray light was measured using a $1 \%$ solution of potassium iodide.

\section{Spectrometer}

The OPT-DISSTM fiber optic spectrometer system was used for all measurements. The spectrometer consisted of a 12-leg source fiber bundle, a 12-leg detector fiber bundle, a shuttered deuterium light source, and a thermo-electrically cooled twodimensional CCD (charge coupled device) array detector coupled to a high throughput $\mathrm{f} / 2$ imaging spectrograph. Individual light beams transmitted through the detector fiber bundle from up to 12 fiber optic probes were imaged through a $0.1 \mathrm{~mm}$ vertical slit onto the CCD detector chip with $512 \mathrm{X}$
512 pixels (light sensitive elements). The systemcontrolled shutter is normally closed to protect source fibers from UV light. The shutter is opened only to expose the CCD to sample light beams for the duration of the integration/exposure time. $\mathrm{A}$ schematic diagram illustrating the system optical train is shown in Figure 1. The spectrometer system (Windows XP/2000 workstation, software, and CCD controller) can simultaneously acquire up to 12 full UV spectra (205 - $410 \mathrm{~nm}$ ) in less than 5 seconds.

\section{Sampling Devices}

Three fiber optic probe types (1- and 10-mm $\mathrm{ARCH}^{\mathrm{TM}}, 10 \mathrm{~mm}$ transflectance) and a $10-\mathrm{mm}$ cuvette were used in the assessment. The ARCH ${ }^{\mathrm{TM}}$ probes (US Patent \#6,580,506, LEAP Technologies, Inc.) are of the transmission type and employ no focusing or reflecting optics. The transflectance probe uses a lens to focus both input and output light beams and a mirror to reflect light back through the sample. The term "transflectance" is used to describe this probe type since transmitted light travels through the sample solution twice due

Figure 1. System optical components: single fiber example

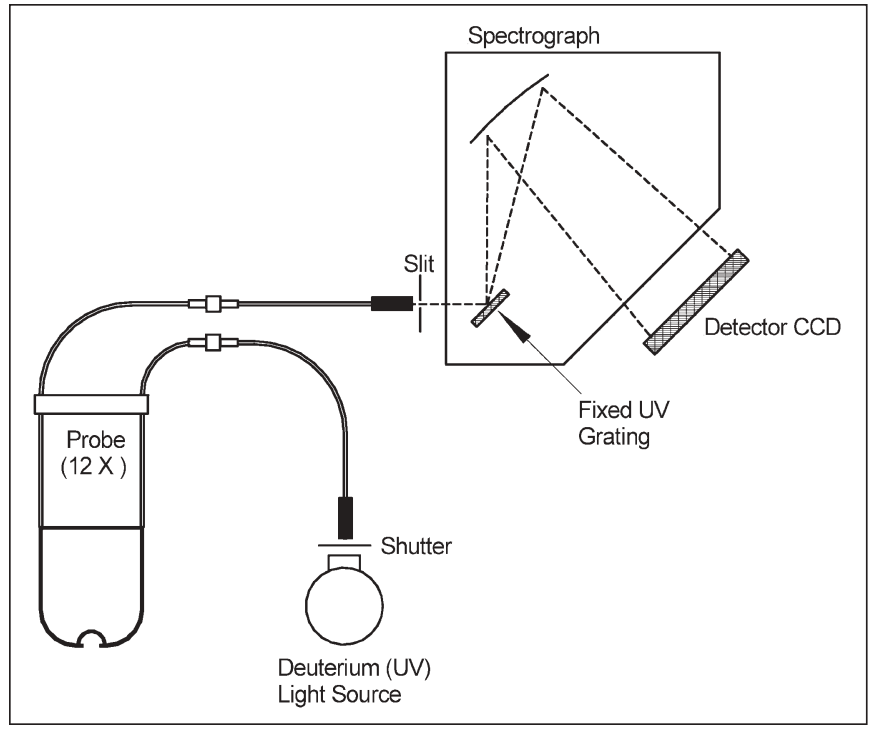


to the reflecting mirror. The cuvette was mounted in a fiber optic cuvette holder equipped with collimating lenses.

\section{Data Acquisition and Processing}

Raw intensity spectra were acquired and absorbance was calculated using OPT-DISSTM software version 1.13. Prior to starting each experiment the detector exposure time was manually optimized to obtain signal strengths of approximately $90 \%$ of detector saturation for the most strongly transmitting probe. This optimization procedure is a required step in preparing the OPT-DISS ${ }^{\mathrm{TM}}$ system for a dissolution test. The total data acquisition time for a complete spectral data set at a given time point is equal to the number of averaged scans X (exposure time + data readout time) where the data readout time was approximately $\sim 0.8 \mathrm{sec}$.

\section{Experimental}

\section{Precision - 12 ARCH $^{\mathrm{TM}}$ Probes}

The system was configured with $1210-\mathrm{mm} \mathrm{ARCH}^{\mathrm{TM}}$ probes immersed in water (theoretical absorbance $=$ 0.0000). Two runs were completed where spectra were acquired at 10-sec and 6-min intervals for total run times of $100 \mathrm{sec}$ and $1 \mathrm{hr}$ respectively. The first run was configured with parameter settings optimized for rapid data collection as would be done to fully characterize active release from a rapidly dissolving dosage form. The second run was configured with parameter settings optimized for data collection for a one-hour dissolution test. For each run the standard deviation of 10 absorbance values was calculated for each of the 12 probes/channels. Mean, low, and high standard deviations at four wavelengths are listed in Tables 1 and 2 .

\section{Precision - Single Sampling Devices}

Runs were completed for 1 - and $10-\mathrm{mm} \mathrm{ARCH}^{\mathrm{TM}}$ probes, a transflectance probe, and a 10-mm cuvette immersed in water. Comparison results are shown in Table 3. Precision at high absorbance values ( $0.75-2.2 \mathrm{AU}$ ) was determined using $1210-\mathrm{mm} \mathrm{ARCH}^{\mathrm{TM}}$ probes immersed in a potassium dichromate solution. Results, as RSD's, are shown in Table 4. In order to assess fundamental instrument performance, absorbance spectra were not smoothed and absorbance values were not corrected for baseline shifts.

\section{Throughput Measurements}

Spectral throughput, Light Output/Light Input X $100 \%$ or $\% \mathrm{~T}$, was determined for each sampling device. Light Input and Output intensities were measured using the "underfilling" fiber connection configurations shown in Figure 2. Spectral throughput plots are shown in Figure 3.

See Quantitative Assessment of Probe ... continued on page 29

\section{Stray Light}

The effect of stray light on system maximum absorbance range was determined using ASTM method E 387 (1) where

\section{Table 1.Standard deviations of 10 absorbance measurements at 10-sec intervals, 12 10-mm ARCH ${ }^{\mathrm{TM}}$ probes in water}

\begin{tabular}{|c|c|c|c|}
\hline \multicolumn{4}{|c|}{$\begin{aligned} \text { Spectra Averaged per Timepoint: } & 4 \\
\text { Integration/Exposure Time: } & 290 \mathrm{~ms} \\
\text { Data Acquisition Time: } & 7 \mathrm{sec} \\
\text { Total Run Time: } & 100 \mathrm{sec} \\
\text { Baseline Correction: } & \text { none } \\
\text { Smoothing: } & \text { none }\end{aligned}$} \\
\hline Wavelength (nm) & Mean & Low & High \\
\hline 215 & 0.0004 & 0.0002 & 0.0006 \\
\hline 250 & 0.0003 & 0.0002 & 0.0004 \\
\hline 300 & 0.0003 & 0.0001 & 0.0005 \\
\hline 350 & 0.0005 & 0.0003 & 0.0008 \\
\hline
\end{tabular}

Table 2. Standard deviations of 10 absorbance measurements at 6-min intervals, 12 10- $\mathrm{mm} \mathrm{ARCH}^{\mathrm{TM}}$ probes in water

\begin{tabular}{|c|c|c|c|}
\hline \multicolumn{4}{|c|}{$\begin{aligned} \text { Spectra Averaged per Timepoint: } & 10 \\
\text { Integration/Exposure Time: } & 275 \mathrm{~ms} \\
\text { Data Acquisition Time: } & 15 \mathrm{sec} \\
\text { Total Run Time: } & 1 \mathrm{hr} \\
\text { Baseline Correction: } & \text { none } \\
\text { Smoothing: } & \text { none }\end{aligned}$} \\
\hline Wavelength $(\mathrm{nm})$ & Mean & Low & High \\
\hline 215 & 0.0003 & 0.0002 & 0.0004 \\
\hline 250 & 0.0002 & 0.0001 & 0.0002 \\
\hline 300 & 0.0002 & 0.0001 & 0.0004 \\
\hline 350 & 0.0003 & 0.0002 & 0.0004 \\
\hline
\end{tabular}

Figure 2. Fiber connections used to measure probe and cuvette throughput

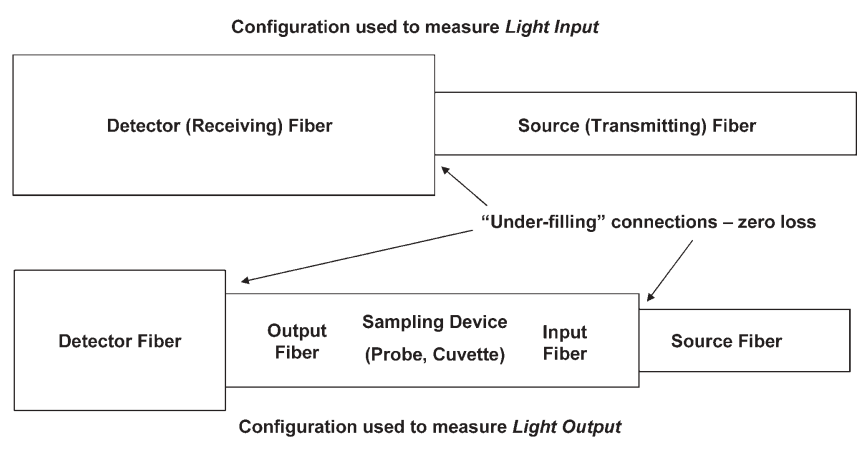




\section{Quantitative Assessment of Probe ... continued}

\section{Table 3. Comparison of precision results (standard deviations of 10 absorbance measurements) for three probe types and a cuvette}

\begin{tabular}{|ccccc|}
\hline \multicolumn{5}{|c|}{ Spectra Averaged per Timepoint: 4} \\
Data Acquisition Time: $10 \mathrm{sec}$ \\
Total Run Time: $100 \mathrm{sec}$ \\
Baseline Correction: none \\
Smoothing: none
\end{tabular}

Table 4. RSDs of 10 absorbance measurements at 6min intervals, $1210-\mathrm{mm} \mathrm{ARCH}^{\mathrm{TM}}$ probes in potassium dichromate solution

\begin{tabular}{|c|c|c|c|c|}
\hline \multicolumn{5}{|c|}{$\begin{aligned} \text { Spectra Averaged perTimepoint: } & 10 \\
\text { Integration/Exposure Time: } & 275 \mathrm{~ms} \\
\text { Data Acquisition Time: } & 15 \mathrm{sec} \\
\text { Total Run Time: } & 1 \mathrm{hr} \\
\text { Baseline Correction: } & \text { none } \\
\text { Smoothing: } & \text { none }\end{aligned}$} \\
\hline $\begin{array}{l}\text { Wavelength } \\
\text { (nm) }\end{array}$ & Absorbance & Mean & Low & High \\
\hline 235 (valley) & 1.94 & 0.17 & 0.14 & 0.25 \\
\hline 257 (peak) & 2.20 & 0.17 & 0.09 & 0.25 \\
\hline 313 (valley) & 0.75 & 0.17 & 0.11 & 0.25 \\
\hline 350 (peak) & 1.60 & 0.21 & 0.16 & 0.30 \\
\hline
\end{tabular}

Table 5. Linearity of absorbance measured using potassium dichromate solutions, 12 10- $\mathrm{mm} \mathrm{ARCH}^{\mathrm{TM}}$ probes: correlation coefficients $\left(r^{2}\right)$ at different wavelengths

\begin{tabular}{|c|c|c|c|}
\hline \multicolumn{4}{|c|}{$\begin{aligned} \text { Absorbance Range: } & 0.0 \text { to } 2.1 \mathrm{AU} \\
\text { Spectra Averaged per Timepoint: } & 4 \\
\text { Integration/Exposure Time: } & 300 \mathrm{~ms} \\
\text { Baseline Correction: } & \text { none } \\
\text { Smoothing: } & \text { none }\end{aligned}$} \\
\hline Wavelength (nm) & Mean & Low & High \\
\hline 235 & 0.9999 & 0.9996 & 1.0000 \\
\hline 257 & 0.9999 & 0.9997 & 0.9999 \\
\hline 350 & 0.9998 & 0.9998 & 0.9999 \\
\hline
\end{tabular}

Figure 3. Spectral throughput curves for different probes and a cuvette

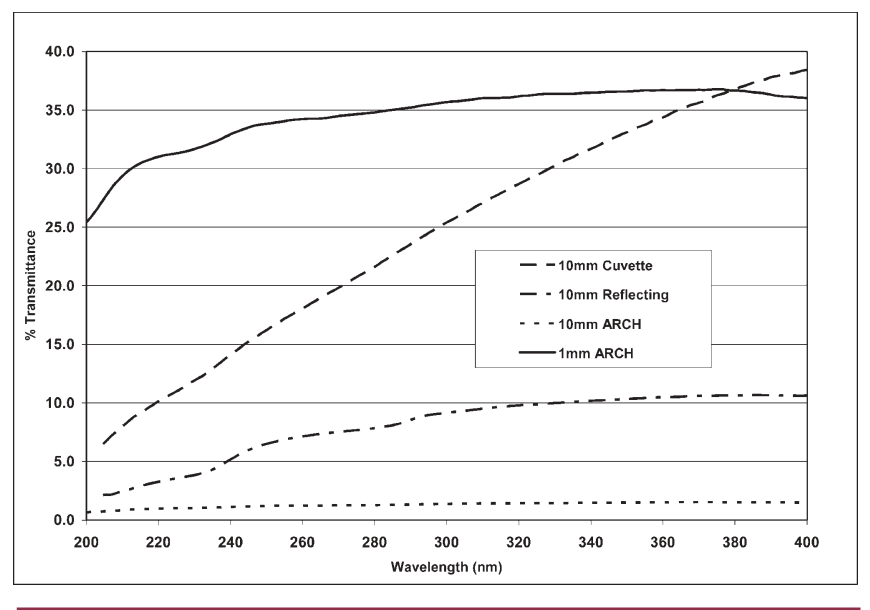

absorbance of an iodide solution is measured at $240 \mathrm{~nm}$. Individual sampling devices were connected to the system and immersed in 1\% potassium iodide. Single absorbance spectra for each sampling device are shown in Figure 4. Spectra for multiple attached probes were acquired for two examples (12 10-mm ARCH probes and six 10-mm transflectance probes) and are shown in Figure 5.

\section{Linearity}

A series of spectra were acquired with $1210-\mathrm{mm} \mathrm{ARCH}$ probes immersed in eight different potassium dichromate solutions ranging in concentration from 0.0 to $0.18 \mathrm{mg} / \mathrm{mL}$ (nominal absorbance of 0.0 - 2.6 AU. Linear regression correlation coefficient results (mean, low, and high $\mathrm{r}^{2}$ ) at different wavelengths are listed in Table 5 . Single linearity spectra were acquired for each sampling device. Comparative linearity plots (absorbance at $257 \mathrm{~nm}$ versus concentration) for each device are shown in Figure 6.

\section{Wavelength Accuracy}

Wavelength accuracy was verified against a NIST primary standard low-pressure mercury vapor lamp. Emission spectra were acquired by connecting the system "source" fiber bundle to the lamp and measuring spectra for $1210-\mathrm{mm}$ ARCH probes immersed in water. Positions of peak maxima for five known bands were determined. Table 6 lists the verification results.

\section{ARCH ${ }^{\mathrm{TM}}$ Probe Hydrodynamic Effects}

Initial hydrodynamic testing of $\mathrm{ARCH}^{\mathrm{TM}}$ probes using USP Lot M Prednisone Tablets is described elsewhere (2). Percent dissolved results using USP Apparatus 2 were determined from samples pulled manually and analyzed off-line from six vessels in five dissolution tests. For each test, three vessels contained no probes and three vessels contained the $\mathrm{ARCH}^{\mathrm{TM}}$ probes. Thus the experimental design yielded 15 


\section{Quantitative Assessment of Probe ... continued}

Figure 4. Maximum absorbance ranges for single probes and a cuvette immersed in $1 \% \mathrm{KI}$

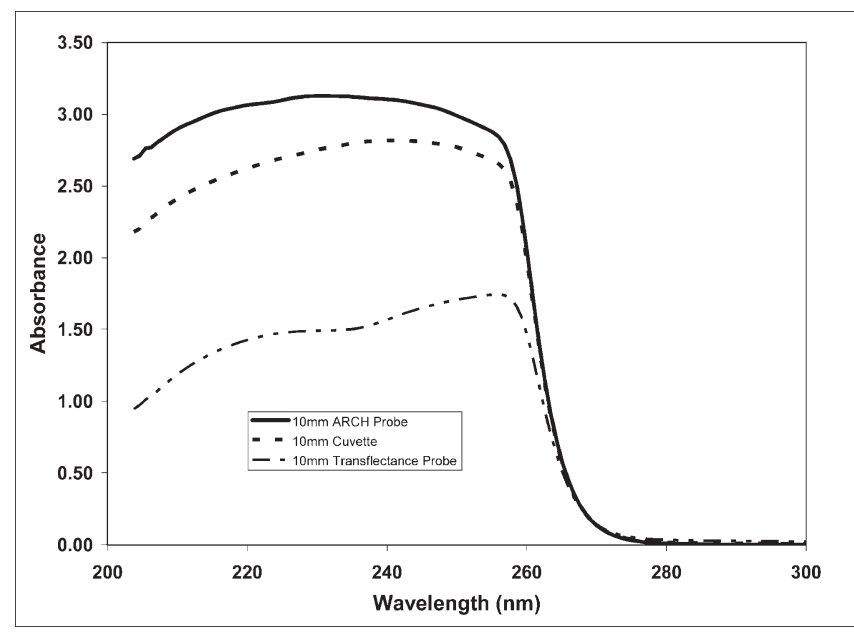

Figure 5. Maximum absorbance ranges for multiple attached $10-\mathrm{mm}$ probes immersed in $1 \% \mathrm{KI}$

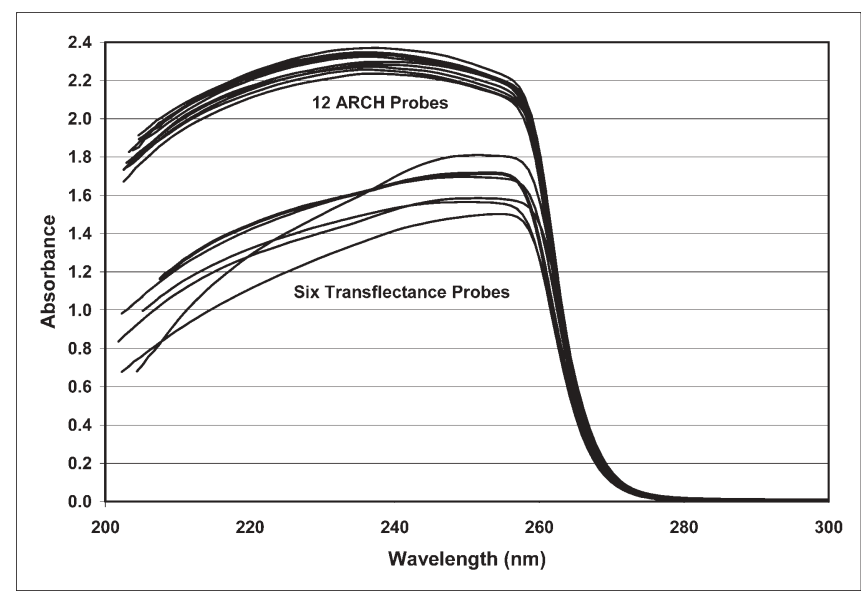

sets of results for each vessel configuration. In this work a new probe design ( $\mathrm{ARCH}{ }^{\mathrm{TM}}$ version 2 ) with a thicker alignment arch was tested, again with Apparatus 2, using USP Lot $\mathrm{N}$ Prednisone Tablets and comparing percent dissolved results for the Version 2 probe against results for the Version 1 probe. Results of both studies are shown in Tables 7 and 8.

\section{Results and Discussion}

\section{Precision, Throughput, and Data Acquisition Time}

Precision experiments focused on 10-mm ARCH ${ }^{\mathrm{TM}}$ probes because of possible concerns related to their low optical throughput (Figure 3). As shown in Tables 1 and 2, the low throughput ( $1-2 \%$ ) had no adverse impact on either the short-term (10-sec interval) or long-tem (6-min) precision results. Precision results are comparable to results for other probe types and a cuvette (Table 3 ). The
Figure 6. Linearity results for $10-\mathrm{mm}$ ARCH probe $\left(r^{2}=0.9999\right), 10-\mathrm{mm}$ transflectance probe $\left(r^{2}=0.962\right)$, and $10-m m$ cuvette $\left(r^{2}=1.0000\right)$.

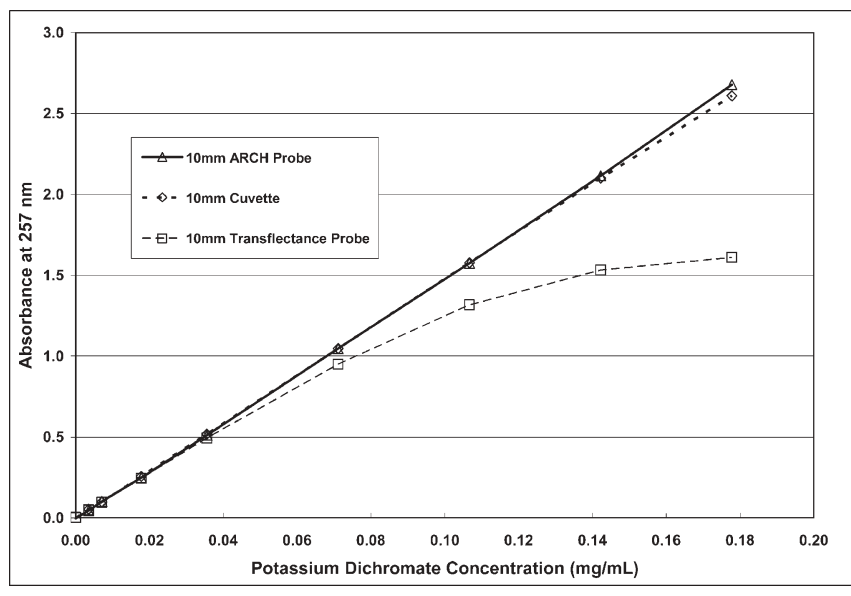

Table 6.Wavelength accuracy results for $1210-\mathrm{mm}$ ARCH ${ }^{\mathrm{TM}}$ probes

\begin{tabular}{|c|c|c|c|c|}
\hline \multirow{2}{*}{$\begin{array}{c}\text { Known } \\
\text { Mercury } \\
\begin{array}{c}\text { Emission } \\
\text { Bands (nm) }\end{array}\end{array}$} & \multicolumn{4}{|l|}{ Observed Peak Maxima Positions (nm) } \\
\cline { 2 - 5 } & Mean & Low & High & $\begin{array}{c}\text { Maximum } \\
\text { Difference }\end{array}$ \\
\hline $\mathbf{2 5 3 . 7}$ & 253.8 & 253.5 & 253.9 & 0.2 \\
\hline $\mathbf{2 9 6 . 7}$ & 296.8 & 296.6 & 297.0 & 0.3 \\
\hline $\mathbf{3 1 3 . 2}$ & 313.1 & 312.9 & 313.3 & -0.3 \\
\hline $\mathbf{3 6 5 . 0}$ & 365.2 & 364.9 & 365.3 & 0.3 \\
\hline $\mathbf{4 0 4 . 7}$ & 404.6 & 404.3 & 404.7 & -0.4 \\
\hline
\end{tabular}

non-dependence of precision results on throughput is attributed to the high sensitivity of the CCD array detector and the fact that the integration/exposure time is optimized prior to a run. Low throughput devices, 10-mm $\mathrm{ARCH} \mathrm{H}^{\mathrm{TM}}$ probes for example, require a longer exposure time (275-300 ms) than do the high throughput devices (8-55 ms). The longer exposure time is required to fill the CCD charge wells to approximately $90 \%$ of their full charge capacity. Since 12 spectra are acquired simultaneously and not sequentially, the longer exposure time, even with four signal-averaged scans, only required a total data acquisition time of $7 \mathrm{sec}$. Precision results for all sampling device types showed no dependence on wavelength over the tested range of $215-350 \mathrm{~nm}$.

\section{Stray Light}

Stray light effects were determined as stray light percentage (\%Stray Light $\left.=100 \times 10^{\text {-Maximum Absorbance }}\right)$ and as the maximum absorbance range. The latter was considered to be a more useful indicator of system performance 
Table 7. Percent dissolved results (for 15 pairs of test results): manual sampling with off-line UV analysis for Lot M Prednisone Tablets using USP Apparatus 2

\begin{tabular}{|ccc|}
\hline & $\begin{array}{c}\text { No Probes } \\
\text { in Vessels }\end{array}$ & $\begin{array}{c}\text { ARCH }^{\mathrm{TM}} \text { Version 1 } \\
\text { Probes in Vessels }\end{array}$ \\
\hline SD: & 1.4 & 1.5 \\
\hline Mean: & 27.4 & 27.9 \\
\hline Range: & $24.6-29.7$ & $25.9-30.8$ \\
\hline
\end{tabular}

Table 8. Percent dissolved results (for 15 pairs of test results): OPT-DISS ${ }^{\mathrm{TM}}$ in situ measurements for Lot $\mathbf{N}$ Prednisone Tablets using USP Apparatus 2

\begin{tabular}{|r|c|c|}
\hline & ARCH $^{\text {TM }}$ Version 1 & ARCH $^{\text {TM }}$ Version 2 \\
\hline SD: & 3.2 & 1.5 \\
\hline Mean: & 36.3 & 35.2 \\
\hline Range: & $32.5-45.6$ & $32.9-39.1$ \\
\hline
\end{tabular}

related to dissolution testing. Results, shown in Figures 4 and 5 , include stray light introduced by the sampling device and the spectrometer. Conventional dip probes with reflecting mirrors and other optical components are known to introduce stray light, limiting the maximum linear absorbance range (3). The primary source of stray light in the OPT-DISSTM spectrometer is light reflected from the quartz window covering the CCD. Stray light arises when a device in the optical train induces harmonic wavelengths of input light. Thus it is possible for light entering the system at one wavelength to be measured at another wavelength. When a single sampling device is connected, the total system stray light is less than when multiple devices are connected. The additional devices add more light and thus increase the amount of stray light. Results in Figure 4 are more indicative of sampling device stray light than those of Figure 5. The ARCH ${ }^{\mathrm{TM}}$ probe exhibits the widest absorbance range $(0.0-3.0 \mathrm{AU})$ and the least amount of stray light $(<0.1 \%)$ of the three devices tested. With $\mathrm{ARCH}^{\mathrm{TM}}$ probes, sample solution is in direct contact with the optical fiber fused silica core. There are no additional optical components (lens, mirror, or cell walls) that can introduce stray light. As shown in Figure 5, when 12 $\mathrm{ARCH}^{\mathrm{TM}}$ probes are attached, the amount of system stray light increases to $0.5 \%$ and the maximum absorbance range decreases to 2.2-2.4 AU. Multiple transflectance probes exhibit a lower maximum absorbance of 1.5-1.8 AU (1.6-3.2\% stray light) than do the $A R C H^{T M}$ probes. The transflectance probes also showed more probe-to-probe variability in stray light and less of a reduction in stray light when connected to the OPT-DISS TM spectrometer.

\section{System Linearity}

System linearity over a given absorbance range and wavelength depends on linearity of detector response and the amount of stray light. The minimal stray light introduced by the $A R C H^{\mathrm{TM}}$ probes helps explain the dichromate system linearity results shown in Table 5 . For a system configured with 12 probes, the correlation coefficients for each channel were 0.9996 or greater over the absorbance range of $0.0-2.1 \mathrm{AU}$ at three different wavelengths. As shown in Figure 6, the linear absorbance range for a single $A R C H^{T M}$ probe is equivalent to that of a cuvette and double the range of a transflectance probe.

\section{Wavelength Accuracy}

Verification of wavelength accuracy for a 12probe/channel system configuration showed that the maximum error was $0.4 \mathrm{~nm}$ at $405 \mathrm{~nm}$ and $0.2-0.3 \mathrm{~nm}$ at lower wavelengths typically used for dissolution analysis. These errors are well within the spectral resolution $(1.7 \mathrm{~nm})$ of the spectrometer and would not adversely affect quantitation using typically broad absorbance quantitation peaks.

\section{Probe Hydrodynamic Effects}

Multiple tests $(n=15)$ using two $A R C H^{T M}$ probe versions showed no impact on percent dissolved results (Tables 7 and 8 ) when the probe remained in place throughout the test. Minor differences in the mean results were well within the observed tablet dissolution variability. Since prednisone disintegrating calibrator tablets are known to be sensitive to hydrodynamic disturbances (4-6) it is probable that $\mathrm{ARCH}$ probes ${ }^{\mathrm{TM}}$ would have minimal or no impact on dissolution hydrodynamics for other dosage forms. Unlike vertical light path dip probes, the $A R C \mathrm{H}^{\mathrm{TM}}$ transverse light path and open structure minimize potential bubble entrapment and particulate buildup during a dissolution test.

\section{Conclusions}

The CCD array spectrometer effectively accommodates different sampling devices that have a wide range of optical throughputs. The high sensitivity of the detector reduces throughput-related design constraints and allows use of probe geometries (the $\mathrm{ARCH}^{\mathrm{TM}}$ for example) that offer advantages over conventional dip probes for dissolution testing. The linear operating range of 0.0-2.1 AU for the OPT-DISSTM/ARCH ${ }^{\mathrm{TM}}$ combination helps meet requirements of dissolution profile testing where drug levels may differ significantly over the duration of a test. The wide linear range extends system applicability to existing methods that would potentially require dilution or a change in probes or probe tips. Rapid and simultaneous full spectral acquisition ensures that USP sampling time 


\section{Quantitative Assessment of Probe ... continued}

constraints are easily met without the need to stagger dropping of dosage forms. The full spectral response allows measurement of active ingredients that absorb in the lower UV range. Absorbance baseline correction at a single reference wavelength is the first-choice technique $(3,7)$ to correct for baseline shifts encountered during in situ testing where filtration is not possible. The system's wide spectral response maximizes the options available for both analytical and reference wavelengths.

\section{References}

1. ASTM Standard Test Method (E 387):"Estimating Stray Radiant Power Ratio of Spectrophotometers by the Opaque Filter Method," ASTM Standards, 14.01, American Society for Testing and Materials, Philadelphia, PA, p. 104 (1993).

2. G. W. Inman et al., "System Optimization for in situ Fiber Optic Dissolution Testing," Pharm. Technol., 25 (10), 92 - 100, October (2001).

3. C. Schatz et al., "Thoughts on Fiber-Optic Dissolution Testing," Dissolution Tech. 8 (2), 6 - 11 (2001).

4. D.C. Cox et al,"Guidelines for Dissolution Testing: An Addendum," Pharm. Technol. 8 (2), 42 - 46 (1984).

5. E. Wells, "Effect of Sampling Probe Size on Dissolution of Tableted Drug Samples," J. Pharm. Sci. 70, 232 (1981).

6. T.S. Savage and C.S. Wells, "Automated Sampling of In Vitro Dissolution Medium: Effect of Sampling Probes on Dissolution Rate of Prednisone Tablets,"J. Pharm. Sci. 71, 670 (1982).

7. C. Schatz et al.,"Evaluation of the Rainbow Dynamic Dissolution Monitor Semi-automatic Fiber Optic Dissolution Tester," Dissolution Tech. 7 (4), 8 - 17 (2000). 